\title{
The prevalence, risk factors and prognostic implications of dysphagia in elderly patients undergoing hip fracture surgery in Korea
}

Seong-Eun Byun ${ }^{1}$, Kyeu Back Kwon², Sang Ho Kim² and Seung-Jae Lim² ${ }^{2^{*}}$

\begin{abstract}
Background: Dysphagia is prevalent in geriatric patients, such as elderly hip fracture patients, and is associated with a poor prognosis. This study investigated (1) the prevalence of dysphagia based on clinical screening and a video-fluoroscopic swallowing study (VFSS), (2) the risk factors of dysphagia, and (3) the prognostic implications of dysphagia in elderly patients ( $\geq 65$ years) undergoing hip fracture surgery.

Methods: In this retrospective study, data from 393 female and 153 male patients $\geq 65$ years of age who underwent surgery for a hip fracture between 2015 and 2018 were analysed. Patients who were considered at high risk of dysphagia after screening underwent a VFSS. To identify risk factors of dysphagia, demographic factors, the American Society of Anesthesiologists classification, past medical history, known risk factors of dysphagia, and factors associated with surgery were analysed using a binary logistic regression model. Odds ratios (ORs) of dysphagia for having poor prognosis including postoperative pneumonia, intensive care unit (ICU) admission, and death within 6 months after surgery were obtained by logistic regression. The association of postoperative pneumonia with poor prognosis was also analysed.

Results: Dysphagia was seen in 5.3\% of hip fracture patients. In multivariate regression analysis, a serum albumin level $<3.5 \mathrm{~g} / \mathrm{dL}$ was identified as a risk factor for dysphagia (OR [95\% $\mathrm{Cl}]=3.13[1.40,7.01])$. Dysphagia was identified as a risk factor for postoperative pneumonia in regression analysis after adjustment (OR $[95 \% \mathrm{Cl}]=3.12[1.05,9.27])$. Postoperative pneumonia was significantly associated with ICU admission $(\mathrm{OR}[95 \% \mathrm{CI}]=4.56[1.85,11.28])$ and death within 6 months after surgery (OR $[95 \% \mathrm{Cl}]=2.56[1.03,6.33])$.

Conclusions: Dysphagia in elderly hip fracture surgery patients was associated with postoperative pneumonia, a risk factor for poor outcomes including ICU admission and death within 6 months after surgery. A serum albumin level $<3.5 \mathrm{~g} / \mathrm{dL}$ was identified as a risk factor for dysphagia. Therefore, diagnostic testing should be performed to detect dysphagia, especially in patients with a low serum albumin level. Finally, particular care should be taken to prevent postoperative complications in patients with dysphagia.
\end{abstract}

Keywords: Dysphagia, Hip fracture, Frailty, Prevalence, Prognosis

\footnotetext{
* Correspondence: limsj70@gmail.com

${ }^{2}$ Department of Orthopaedic Surgery, Samsung Medical Center,

Sungkyunkwan University School of Medicine, 81 Irwon-ro, Gangnam-gu,

Seoul 06351, Republic of Korea

Full list of author information is available at the end of the article
}

(c) The Author(s). 2019 Open Access This article is distributed under the terms of the Creative Commons Attribution 4.0 International License (http://creativecommons.org/licenses/by/4.0/), which permits unrestricted use, distribution, and reproduction in any medium, provided you give appropriate credit to the original author(s) and the source, provide a link to the Creative Commons license, and indicate if changes were made. The Creative Commons Public Domain Dedication waiver (http://creativecommons.org/publicdomain/zero/1.0/) applies to the data made available in this article, unless otherwise stated. 


\section{Background}

Dysphagia, or disordered oropharyngeal swallowing, is a common finding in frail elderly patients caused by agerelated physiological changes in swallowing, including a reduced neuronal coordination of the swallowing process, and decreased digestive tract motility due to decreases in muscle mass and connective tissue elasticity [1-3]. Dysphagia may lead to malnutrition, dehydration, and aspiration, which is the misdirection of oropharyngeal contents into the respiratory tract [3]. Aspiration in frail elderly patients can lead to aspiration pneumonia, a common and fatal postoperative complication. Therefore, a relationship of dysphagia with increases in mortality and length of hospital stay has been identified in hospitalized patients [4].

Osteoporotic hip fracture is a major health problem due to its association with a high mortality rate and its negative influence on the quality of life $[5,6]$. Moreover, as populations age, osteoporotic hip fracture patients become more common [7]. Elderly patients with hip fractures are usually those who are frail and often present with an impaired functional status across several health metrics $[8,9]$. In frail patients, an acute illness or procedure, including hip fracture surgery, may worsen an already reduced functional reserve and thus increase patient vulnerability to dysphagia [10]. A previous report identified a high prevalence of dysphagia in hip fracture patients [11]. A negative impact of aspiration pneumonia, which is closely related to dysphagia, on the prognosis of hip fracture patients has also been noted [12] Therefore, the detection of dysphagia is an important factor in determining the prognosis of elderly patients with hip fracture.

Although a video-fluoroscopic swallowing study (VFSS) is considered the gold standard for diagnosing dysphagia, previous studies of dysphagia in hip fracture patients have relied on clinical methods to obtain a diagnosis [12, 13, 14]. Therefore, in the present study, we investigated (1) the prevalence of dysphagia using clinical screening and VFSS, (2) the risk factors for dysphagia, and (3) the prognostic implications of dysphagia in elderly ( $\geq 65$ years) patients undergoing hip fracture surgery.

\section{Methods}

This study was conducted with the approval of the Institutional Review Board. Informed consent was waived due to the retrospective design of the study. A total of 570 patients $\geq 65$ years of age who underwent surgery for hip fracture (femoral neck fracture and intertrochanteric fracture) at the senior author's institution (tertiary referral hospital) in Seoul, Republic of Korea between March 2015 and May 2018 were retrospectively analysed. Patients Cases of ipsilateral or contralateral hip fracture dislocation (1 patient), acetabular fracture or pelvic ring injury (3 patients), and femoral shaft fracture (1 patient) were not included in this study. Cases of pathologic fracture caused by neoplasms (11 patients) were also excluded, as were those presenting with pneumonia at the time of admission (7 patients). In addition, one patient whose medical records were insufficient for the analysis was not included. Therefore, 546 hip fracture patients (274 femoral neck fractures and 272 intertrochanteric fractures) constituted the study cohort.

\section{Diagnosis of dysphagia}

Beginning in March 2015, the institution where the current study was performed implemented a protocol to detect oropharyngeal dysphagia and prevent postoperative pneumonia. Patients were screened for dysphagia at the time of admission to the ward. Thus, patients with a history of aspiration pneumonia or who complained of symptoms of aspiration, such as coughing or hoarseness during a meal, or with abnormalities (choking or difficulty drinking) in a water swallowing test using $30 \mathrm{~mL}$ of water at normal temperature [15], were considered at high risk of dysphagia and underwent a VFSS.

VFSS was performed by an experienced rehabilitation physician, assisted by a radiologic technician, in the fluoroscopic laboratory immediately after a patient assigned to the high-risk dysphagia group. Various boluses $(3,6$, and $9 \mathrm{~mL})$ were used in this study. Initially, a thick, curd-type yogurt was given to the patient. If there no dysphagia occurred, semisolid rice gruel was provided. The patient then received boiled rice in solid and liquid forms. Finally, $30 \mathrm{~mL}$ of diluted barium was provided. Patients were diagnosed as dysphagic if they showed any of the following abnormalities: 1) impaired mastication, lingual motion, or lip closure during the oral phase; 2) decreased pharyngeal contraction or swallowing reflex during the pharyngeal phase; 3) decreased laryngeal contraction, decreased laryngeal closure, or epiglottic inversion during the laryngeal phase; or 4) upper oesophageal sphincter dysfunction or decreased oesophageal contraction.

\section{Analysis of risk factors}

The following demographic data were recorded: age, gender, body mass index (BMI), and indicators of the general condition of the patient, including the American Society of Anesthesiologists (ASA) classification. The medical histories of the patients, including stroke, dementia, and smoking, were assessed together with surgery-related factors (duration of the operation, interval between injury and surgery, method of anaesthesia, and surgical technique).

Results of blood chemistry tests, including albumin, blood urea nitrogen (BUN), and creatinine levels at admission, were reviewed. Blood was collected just after the fracture was confirmed in the emergency department. The serum albumin level was used as a marker of 
malnutrition, with a cut-off value of $3.5 \mathrm{~g} / \mathrm{dL}$. The BUN/ creatinine ratio served as a marker for dehydration, with a cut-off value of 20:1.

\section{Prognostic implications of dysphagia}

The prognostic implications of dysphagia were assessed based on a review of the patient's medical records; specifically, the frequency of postoperative pneumonia, admission to the intensive care unit (ICU), death within 6 months after surgery. Postoperative pneumonia was diagnosed by radiologic study, including chest radiographs and computed tomography, and from the medical records, including consultations with a pulmonologist. Death within 6 months after surgery could be determined by reference to the end date of national health insurance for each patient.

\section{Statistical analysis}

Categorical variables were compared using the chisquared test. The chi-square test had 1 degree of freedom, since all categorical variables had two categories. Continuous variables were compared between groups using the Student's t-test. The t-test had 544 degrees of freedom, since the number of patients included in the analysis was 546. The risk factors for dysphagia were analysed using a binary logistic regression model. Odds ratios (ORs) with 95\% confidence intervals (CIs) were calculated.

A binary logistic regression analysis was performed to evaluate the association of dysphagia with postoperative pneumonia, ICU admission, and death within 6 months after surgery. The correlation of postoperative pneumonia, which is closely associated with dysphagia, with ICU admission and death within 6 months after surgery was also analysed.

A $P$-value $<0.05$ was considered to indicate statistical significance. SPSS version 24.0 (IBM Corp., Armonk, NY) was used for statistical analyses.

\section{Results}

Among the 546 patients, VFSS was performed in 40 patients $(7.3 \%)$ who were considered to be at high risk for dysphagia, and dysphagia was diagnosed in $72.5 \%$ (29 of 40) of the patients. Thus, the prevalence of dysphagia among 546 elderly patients undergoing hip fracture surgery was $5.3 \%$ in our analysis.

Among the 29 patients with dysphagia, there were 14 males and 15 females with a mean age of 82.3 years (range: 72-95 years). Among patients without dysphagia, there were 139 males and 378 females with a mean age of 80.1 years (range: 64-104 years). The demographic data and risk factors of the two groups are summarized in Table 1.

Male gender, BMI, ASA classification $\geq 3$, history of stroke, smoking, and serum albumin level $<3.5 \mathrm{~g} / \mathrm{dL}$ were significant risk factors for dysphagia in the regression analysis without adjustment and were thus included in the multivariate regression model. Multivariate analysis showed that a serum albumin level $<3.5 \mathrm{~g} / \mathrm{dL}$ was an independent risk factor for dysphagia $(\mathrm{OR}=3.13$; $95 \%$ $\mathrm{CI}=1.40-7.01, P=0.005)$ (Table 2).

The associations of dysphagia and postoperative pneumonia with poor outcomes are shown in Table 3. After adjusting for age and gender (model 1), dysphagia showed a significant correlation with postoperative pneumonia and ICU admission. However, after adding variables regarding general condition (model 2) and mental status (model 3) as covariates, dysphagia was significantly related only to postoperative pneumonia dysphagia. Postoperative pneumonia was significantly associated with ICU admission and death within 6 months after surgery in all models.

\section{Discussion}

In our study, dysphagia was diagnosed in $5.3 \%$ of 546 hip fracture surgery patients $\geq 65$ years of age. A low serum albumin level was identified as an independent risk factor for dysphagia. Dysphagia was significantly associated with postoperative pneumonia, which is a risk factor for ICU admission and death within 6 months of surgery.

The prevalence of dysphagia has been studied in various patient populations using different diagnostic methods $[4,11,16]$. In the study of Altman et al. [4], dysphagia was diagnosed in only $0.73 \%$ of hospitalized patients over 75 years of age based on a retrospective analysis of data from the National Hospital Discharge Survey. Studies based on a clinical diagnosis of dysphagia reported higher prevalence rates. For example, Cichero et al. [16] reported a $25-30 \%$ prevalence among acutely hospitalized patients who underwent clinical screening for dysphagia. In their study of dysphagia in hip fracture patients, Love et al. [11] reported a prevalence rate of $34 \%$ among patients $\geq 65$ years of age based on clinical diagnosis by a speech pathologist. In our study, $7.3 \%$ of hip fracture patients were considered to be at risk of dysphagia and thus underwent VFSS, which is considered the gold standard in the diagnosis of dysphagia [13]. Among those who were screened, $72.5 \%$ (5.3\% of all hip fracture patients) were diagnosed with dysphagia. However, dysphagia may have been underdiagnosed, since not all hip fracture patients underwent a VFSS.

In the logistic regression analysis performed in this study, malnutrition, defined as a serum albumin level $<$ $3.5 \mathrm{~g} / \mathrm{dL}$, was significantly associated with dysphagia. Malnutrition has been considered a sequela of dysphagia, since impaired swallowing can interfere with food intake [14]. On the other hand, malnutrition can also cause dysphagia via its negative effects on muscles and nerves [15]. Malnutrition has been suggested as one of the major factors promoting sarcopenia, as well as 
Table 1 Demographic data and risk factors of dysphagia in hip fracture patients $\geq 65$ years of age

\begin{tabular}{|c|c|c|c|c|c|c|}
\hline \multirow[t]{2}{*}{ Variable } & $\begin{array}{l}\text { Total } \\
(N=546)\end{array}$ & $\begin{array}{l}\text { Dysphagia } \\
(n=29)\end{array}$ & $\begin{array}{l}\text { Non-dysphagic } \\
(n=517)\end{array}$ & & & \\
\hline & Mean (SD) or $n(\%)$ & Mean (SD) or $n(\%)$ & Mean (SD) or $n(\%)$ & Chi-square & Effect size & $P$ \\
\hline Age (years) & $80.3(7.0)$ & $82.3(6.0)$ & $80.1(7.0)$ & & 0.307 & 0.109 \\
\hline Gender (male/female) & $153 / 393$ & $14 / 15$ & $139 / 378$ & 6.229 & 0.108 & 0.013 \\
\hline BMI $\left(\mathrm{kg} / \mathrm{m}^{2}\right)$ & $22.1(4.3)$ & $20.6(3.6)$ & $22.2(4.3)$ & & 0.370 & 0.053 \\
\hline ASA classification $\geq 3$ & 295 (54.0\%) & $23(79.3 \%)$ & $272(52.6 \%)$ & 7.881 & 0.120 & 0.005 \\
\hline Stroke & $90(16.5 \%)$ & $9(31.0 \%)$ & $81(15.7 \%)$ & 4.710 & 0.093 & 0.030 \\
\hline Dementia & $86(15.8 \%)$ & $8(27.6 \%)$ & $78(15.1 \%)$ & 3.233 & 0.077 & 0.072 \\
\hline Delirium & $38(7.0 \%)$ & $5(17.2 \%)$ & $33(6.4 \%)$ & 5.000 & 0.096 & 0.025 \\
\hline Smoking & $28(5.1 \%)$ & $5(17.2 \%)$ & $23(4.4 \%)$ & 9.852 & 0.134 & 0.002 \\
\hline Fracture type & & & & 1.731 & 0.056 & 0.188 \\
\hline Femoral neck & $274(50.2 \%)$ & $18(62.1 \%)$ & $256(49.5 \%)$ & & & \\
\hline Intertrochanteric & $272(49.8 \%)$ & $11(37.9 \%)$ & $261(50.5 \%)$ & & & \\
\hline Operation time (min) & $110.6(24.1)$ & $116.8(39.4)$ & $109.7(22.9)$ & & 0.298 & 0.122 \\
\hline Interval between injury and operation $\geq 2$ days & $70(12.8 \%)$ & $7(24.1 \%)$ & $63(12.2 \%)$ & 3.510 & 0.080 & 0.061 \\
\hline Method of anaesthesia & & & & 0.680 & 0.035 & 0.410 \\
\hline General & $242(44.3 \%)$ & $15(51.7 \%)$ & $227(43.9 \%)$ & & & \\
\hline Spinal & $304(55.7 \%)$ & $14(48.3 \%)$ & $290(56.1 \%)$ & & & \\
\hline Surgical technique & & & & 2.976 & 0.074 & 0.084 \\
\hline Arthroplasty & $254(46.5 \%)$ & $18(62.1 \%)$ & $236(45.6 \%)$ & & & \\
\hline Internal fixation & $292(53.5 \%)$ & $11(37.9 \%)$ & $281(54.4 \%)$ & & & \\
\hline Malnutrition (Albumin<3.5 g/dL) & $110(20.1 \%)$ & $14(48.3 \%)$ & $96(18.6 \%)$ & 15.673 & 0.120 & 0.005 \\
\hline Dehydration (BUN/creatinine > 20) & $355(65.0 \%)$ & $21(72.4 \%)$ & $334(64.6 \%)$ & 0.958 & 0.042 & 0.391 \\
\hline
\end{tabular}

Abbreviations: BMI body mass index, ASA American Society of Anesthesiologists; BUN, blood urea nitrogen

$P=$ Differences between patients with and without dysphagia

To analyse categorical variables, the Chi square test was performed and the phi coefficient was calculated for the effect size

To analyse continuous variables, te t-test was used and Cohen's d was calculated for the effect size

Table 2 Multivariate analysis of the risk factors for dysphagia in hip fracture patients $\geq 65$ years of age

\begin{tabular}{lllll}
\hline Variable & Analytic model & Odds ratio & $95 \% \mathrm{Cl}$ & $P$ \\
\hline Gender (male) & Model 1 & 2.54 & $1.19-5.39$ & 0.015 \\
& Model 2 & 1.43 & $0.61-3.34$ & 0.414 \\
BMl & Model 1 & 0.90 & $0.81-1.00$ & 0.045 \\
& Model 2 & 0.96 & $0.86-1.07$ & 0.427 \\
ASA classification $\geq 3$ & Model 1 & 3.45 & $1.38-8.62$ & 0.008 \\
& Model 2 & 2.19 & $0.83-5.76$ & 0.113 \\
Stroke & Model 1 & 2.42 & $1.07-5.51$ & 0.035 \\
& Model 2 & 1.81 & $0.75-4.39$ & 0.188 \\
Smoking & Model 1 & 4.69 & $1.63-13.45$ & 0.004 \\
& Model 2 & 2.91 & $0.86-9.80$ & 0.085 \\
Albumin <3.5 g/dL & Model 1 & 4.20 & $1.96-9.00$ & $<0.001$ \\
& Model 2 & 3.13 & $1.40-7.01$ & 0.005 \\
\hline
\end{tabular}

Abbreviations: $C l$ confidence interval, $B M I$ body mass index, ASA American Society Anesthesiologists

1. Model 1 was not adjusted

2. Model 2 included all six variables listed in the table decreased muscle mass and strength [17]. In addition, changes in nerve function, including a reduction of nerve conduction velocity in patients with malnutrition, has also been noted [18]. An association between decreased systemic muscle mass and reduced swallowing function has been reported $[19,20]$, where a wellcoordinated nervous system is essential to harmonize the various muscle actions involved in the swallowing process [21]. Accordingly, a circular relationship between poor nutritional status, neuromuscular dysfunction, and dysphagia was suggested [22]. Malnutrition is both a risk factor for, and a consequence of, dysphagia.

Our results demonstrate the negative implications of dysphagia with respect to the prognosis of hip fracture patients. Previous studies also reported poor prognosis in patients with dysphagia $[4,23]$. In the above-cited study of Altman et al. [4], hospitalized patients with dysphagia had a higher rate of aspiration pneumonia, a longer hospitalization, and a higher proportion of death. Moreover, Cabre et al. [23] reported a higher proportion of death in pneumonia patients with dysphagia. Several 
Table 3 Association of dysphagia and postoperative pneumonia with poor prognosis in hip fracture patients $\geq 65$ years of age

\begin{tabular}{|c|c|c|c|c|}
\hline Variables & Analytic model & Odds ratio & $95 \% \mathrm{Cl}$ & $P$ \\
\hline \multicolumn{5}{|l|}{ Dysphagia } \\
\hline \multirow[t]{3}{*}{ Postoperative pneumonia } & Model 1 & 4.52 & $1.64-12.48$ & 0.004 \\
\hline & Model 2 & 3.19 & $1.10-9.21$ & 0.032 \\
\hline & Model 3 & 3.12 & $1.05-9.27$ & 0.041 \\
\hline \multirow[t]{3}{*}{ ICU admission } & Model 1 & 2.34 & $1.02-5.36$ & 0.045 \\
\hline & Model 2 & 1.48 & $0.61-3.59$ & 0.392 \\
\hline & Model 3 & 1.55 & $0.62-3.88$ & 0.352 \\
\hline \multirow[t]{3}{*}{ Death $^{a}$} & Model 1 & 2.00 & $0.79-5.03$ & 0.141 \\
\hline & Model 2 & 1.23 & $0.46-3.27$ & 0.683 \\
\hline & Model 3 & 1.20 & $0.44-3.23$ & 0.724 \\
\hline \multicolumn{5}{|l|}{ Postoperative pneumonia } \\
\hline \multirow[t]{3}{*}{ ICU admission } & Model 1 & 5.59 & $2.52-12.42$ & $<0.001$ \\
\hline & Model 2 & 3.60 & $1.51-8.59$ & 0.004 \\
\hline & Model 3 & 4.56 & $1.85-11.28$ & 0.001 \\
\hline \multirow[t]{3}{*}{ Death $^{a}$} & Model 1 & 3.73 & $1.60-8.70$ & 0.002 \\
\hline & Model 2 & 2.50 & $1.02-6.16$ & 0.046 \\
\hline & Model 3 & 2.56 & $1.03-6.33$ & 0.042 \\
\hline
\end{tabular}

Abbreviations: $\mathrm{Cl}$ confidence interval, BMI body mass index, ASA American Society Anesthesiologists, ICU intensive care unit

Variables included in the model were based on univariate analysis

Notes: ${ }^{\text {a }}$ death within 6 months after surgery

1. Model 1 was adjusted for age and gender

2. Model 2 was adjusted for age, gender, BMI, ASA classification $\geq 3$, and albumin level $<3.5 \mathrm{~g} / \mathrm{dL}$

3. Model 3 was adjusted for age, gender, BMl, ASA classification $\geq 3$, albumin level $<3.5 \mathrm{~g} / \mathrm{dL}$, dementia, delirium, and method of anaesthesia

factors are associated with a worse prognosis for hip fracture patients with dysphagia. First, dysphagia is closely associated with pneumonia and aspiration pneumonia [1]. In hip fracture patients, pneumonia is the most common postoperative complication [24], and is associated with poor outcomes, including longer hospital stay and higher mortality $[12,25]$. In the current study, dysphagia was a risk factor for postoperative pneumonia, which was in turn found to be a risk factor for ICU admission and death within 6 months after surgery. Second, as confirmed in this study, dysphagia is closely associated with malnutrition [22], which, in hip fracture patients, is a risk factor for a poor prognosis [26, 27]. Drevet et al. [27] reported that hip fracture patients with protein-energy malnutrition had longer hospital stays. In their study of hip fracture patients, Chung et al. [26] showed that malnutrition, assessed by measuring the serum albumin level, was associated with postoperative complications, a longer hospital stay, and a higher mortality rate. The negative impact on prognosis of malnutrition may be due to its association with frailty and a reduced physiologic reserve, as also seen under conditions of surgical stress, for example [28, 29]. Third, and related to the second reason, dysphagia is not a distinct disorder of the oropharynx, but rather one of the phenotypes of frailty syndrome, which includes sarcopenia.
Therefore, patients with dysphagia are likely to have comorbidities or other conditions affecting their prognosis. Singh et al. [30] demonstrated that, in patients who underwent spinal surgery, dysphagia was associated with age $>$ 65 years, weight loss, and comorbidities including anaemia, neurological disorders, solid tumours, and fluid/ electrical disorders. Maeda and Akagi [31] also showed that a low skeletal muscle mass and poor performance status were associated with dysphagia. In our study, patients with dysphagia had a higher ASA classification grade, and thus a larger number of comorbidities, as well as a poorer general condition than those without dysphagia.

There were several limitations to the current study. First, VFSS, a diagnostic study for oropharyngeal dysphagia, was not performed in all patients. Although all patients were screened following a VFSS, the screening method used may not have been able to detect patients with silent aspiration [32]. Therefore, the reported prevalence of dysphagia may have been lower than the actual prevalence, where this bias could have affected the results. Second, since our study was retrospective and included patients who were operated on at a referral hospital, selection bias may have affected the results. Third, the case-control study design used for identifying risk factors of dysphagia did not allow for the detection of causal relationships. Lastly, the serum albumin level 
may not represent the nutritional status of patients with inflammatory stress [33]. The Mini Nutritional Assessment would be preferable for assessing nutritional status. However, serum albumin level was used for this retrospective study since those data were available; moreover, it has been noted as a risk factor for poor outcomes in hip fracture patients [26, 34].

Despite these limitations, a strength of this study was that it assessed not only the prevalence but also the prognostic implications of dysphagia in elderly patients with hip fracture. In addition, the diagnosis was made using VFSS, the current gold standard for the diagnosis of dysphagia [13].

\section{Conclusion}

Dysphagia in elderly hip fracture surgery patients was associated with postoperative pneumonia, a known risk factor for poor outcomes including ICU admission and death within 6 months after surgery. A serum albumin level $<3.5 \mathrm{~g} / \mathrm{dL}$ was identified as an independent risk factor for dysphagia. Therefore, diagnostic testing should be performed to detect dysphagia, especially in patients with low serum albumin levels. Finally, particular care should be taken to prevent postoperative complications in patients with dysphagia.

\section{Abbreviations}

ASA: American Society of Anesthesiologists; BMI: Body mass index; BUN: Blood urea nitrogen; Cl: Confidence intervals; ICU: Intensive care unit; OR: Odds ratio; VFSS: Video-fluoroscopic swallowing study

\section{Acknowledgements}

Not applicable.

\section{Authors' contribution}

SB: conception and design of the study, analysis and interpretation of the data and writing of the manuscript. KK: conception and design of the study and acquisition and analysis of the data. SK: conception and design of the study and acquisition and analysis of the data. SL: conception and design of the study, writing of the manuscript, and revising manuscript critically for important intellectual content. All authors have read and approved the final manuscript.

\section{Funding}

The authors received no specific funding for this work.

\section{Availability of data and materials}

The datasets used and/or analysed during the current study are available from the corresponding author on reasonable request.

\section{Ethics approval and consent to participate}

The study design was approved by the institutional review board of Samsung Medical Center (SMC IRB, Number: SMC 2018-11-148-001) and the requirement for informed consent was waived due to the retrospective nature of the study.

\section{Consent for publication}

Not applicable.

\section{Competing interests}

The authors declare that they have no competing interests.

\section{Author details}

${ }^{1}$ Department of Orthopaedic Surgery, CHA Bundang Medical Center, CHA University, Seongnam-si, Gyeonggi-do, Republic of Korea. ${ }^{2}$ Department of Orthopaedic Surgery, Samsung Medical Center, Sungkyunkwan University School of Medicine, 81 Irwon-ro, Gangnam-gu, Seoul 06351, Republic of Korea.

Received: 28 January 2019 Accepted: 11 December 2019

Published online: 18 December 2019

\section{References}

1. Marik P, Kaplan D. Aspiration pneumonia and dysphagia in the elderly. Chest. 2003;124:328-36.

2. Fucile S, Wright PM, Chan I, Yee S, Langlais ME, Gisel EG. Functional oralmotor skills: do they change with age? Dysphagia. 1998;13:195-201.

3. Ebihara S, Sekiya H, Miyagi M, Ebihara T, Okazaki T. Dysphagia, dystussia, and aspiration pneumonia in elderly people. J Thorac Dis. 2016;8:632-9.

4. Altman K, Yu G, Schaefer S. Consequence of dysphagia in the hospitalized patient. Arch Otolaryngol Head Neck Surg. 2010;136:784-9.

5. Dyer SM, Crotty M, Fairhall N, et al. A critical review of the long-term disability outcomes following hip fracture. BMC Geriatr. 2016;16:158.

6. Kanis JA, Oden A, Johnell O, De Laet C, Jonsson B, Oglesby AK. The components of excess mortality after hip fracture. Bone. 2003;32:468-73.

7. Lee YK, Kim JW, Lee MH, Moon KH, Koo KH. Trend in the age-adjusted incidence of hip fractures in South Korea: systematic review. Clin Orthop Surg. 2017;9:420-3.

8. Rolland Y, Abellan van Kan G, Bénétos A, et al. Frailty, osteoporosis and hip fracture: causes, consequences and therapeutic perspectives. J Nutr Health Aging. 2008;12:335-46.

9. Liu LK, Lee WJ, Chen LY, et al. Association between frailty, osteoporosis, falls and hip fractures among community-dwelling people aged 50 years and older in Taiwan: results from I-Lan longitudinal aging study. PLoS One. 2015; 10:1-12.

10. Ney DM, Weiss JM, Kind AJ, Robbins J. Senescent swallowing: impact, strategies and interventions. Nutr Clin Pract. 2010;24:395-413.

11. Love AL, Cornwell PL, Whitehouse SL. Oropharyngeal dysphagia in an elderly post-operative hip fracture population: a prospective cohort study. Age Ageing. 2013;42:782-5.

12. Byun S-E, Shon H-C, Kim JW, Kim HK, Sim Y. Risk factors and prognostic implications of aspiration pneumonia in older hip fracture patients : a multicenter retrospective analysis. Geriatr Gerontol Int. 2018. https://doi.org/ 10.1111/ggi.13559.

13. Logemann JA, Veis S, Colangelo L. A screening procedure for oropharyngeal dysphagia. Dysphagia. 1999;14:44-51.

14. Veldee MS, Peth LD. Can protein-calorie malnutrition cause dysphagia? Dysphagia. 1992;7:86-101.

15. McGinnis CM, Homan K, Solomon M, Taylor J, Staebell K, Erger D, Raut N. Dysphagia: Interprofessional Management, Impact, and Patient-Centered Care. Nutr Clin Pract. 2019;34:80-95.

16. Cichero JA, Heaton S, Bassett L. Triaging dysphagia: nurse screening for dysphagia in an acute hospital. J Clin Nurs. 2009;18:1649-59.

17. Cruz-Jentoft AJ, Baeyens JP, Bauer JM, et al. Sarcopenia: European consensus on definition and diagnosis. Age Ageing. 2010;39:412-23.

18. Chopra JS, Dhand UK, Mehta S, Bakshi V, Rana S, Mehta J. Effect of protein calorie malnutrition on peripheral nerves: a clinical, electrophysiological and histopathological study. Brain. 1986;109:307-23.

19. Murakami M, Hirano H, Watanabe Y, Sakai K, Kim H, Katakura A. Relationship between chewing ability and sarcopenia in Japanese community-dwelling older adults. Geriatr Gerontol Int 2015;15:1007-12.

20. Kuroda Y, Kuroda R. Relationship between thinness and swallowing function in Japanese older adults: implications for sarcopenic dysphagia. J Am Geriatr Soc. 2012;60:1785-6.

21. Saito T, Hayashi K, Nakazawa H, Ota T. Clinical characteristics and lesions responsible for swallowing hesitation after acute cerebral infarction. Dysphagia. 2016:31:567-73.

22. Saito T, Hayashi K, Nakazawa H, Yagihashi F, Oikawa LO, Ota T. A significant association of malnutrition with dysphagia in acute patients. Dysphagia. 2018:33:258-65.

23. Cabre M, Serra-Prat M, Palomera E, Almirall J, Pallares R, Clavé P. Prevalence and prognostic implications of dysphagia in elderly patients with pneumonia. Age Ageing. 2009;39:39-45. 
24. Belmont PJ Jr, Garcia EJ, Romano D, Bader JO, Nelson KJ, Schoenfeld AJ. Risk factors for complications and in - hospital mortality following hip fractures: a study using the National Trauma Data Bank. Arch Orthop Trauma Surg. 2014;134:597-604.

25. von Friesendorff M, McGuigan FE, Wizert A, et al. Hip fracture, mortality risk, and cause of death over two decades. Osteoporos Int. 2016;27:2945-53.

26. Chung AS, Hustedt JW, Walker R, Jones C, Lowe J, Russell GV. Increasing severity of malnutrition is associated with poorer 30-day outcomes in patients undergoing hip fracture surgery. J Orthop Trauma. 2018;32:155-60.

27. Drevet $\mathrm{S}$, Bioteau $\mathrm{C}$, Mazière $\mathrm{S}$, et al. Prevalence of protein-energy malnutrition in hospital patients over 75 years of age admitted for hip fracture. Orthop Traumatol Surg Res. 2014;100:669-74.

28. Boulos C, Salameh P, Barberger-gateau P. Malnutrition and frailty in community dwelling older adults living in a rural setting. Clin Nutr. 2016;35:138-43.

29. Wei K, Shwe M, Nyunt Z, Gao Q, Liang S. Frailty and malnutrition : related and distinct syndrome prevalence and association among communitydwelling older adults : Singapore longitudinal ageing studies. J Am Med Dir Assoc. 2017;18:1019-28.

30. Singh K, Marquez-Lara A, Nandyala SV, Patel AA, Fineberg SJ. Incidence and risk factors for dysphagia after anterior cervical fusion. Spine. 2013;38:1820-5.

31. Maeda K, Akagi J. Sarcopenia is an independent risk factor of dysphagia in hospitalized older people. Geriatr Gerontol Int. 2016;16:515-21.

32. Lim SH, Lieu PK, Phua SY, Seshadri R, Venketasubramanian N, Lee SH, Choo PW. Accuracy of bedside clinical methods compared with fiberobtic endoscopic examination of swallowing (FEES) in determining the risk of aspiration in acute stroke patients. Dysphagia. 2001;16:1-6.

33. Moshage HJ, Janssen J, Franssen J, Hafkenschied JC, Yap SH. Study of the molecular mechanism of decreased liver synthesis of albumin in inflammation. J Clin Invest. 1987;79:1635-41.

34. Bohl DD, Shen MR, Hanon CP, Fillingham YA, Darrith B, Della Valle CJ. Serum albumin predicts survival and postoperative course following surgery for geriatric hip fracture. J Bone Joint Surg Am. 2017;99:2110-8.

\section{Publisher's Note}

Springer Nature remains neutral with regard to jurisdictional claims in published maps and institutional affiliations.

Ready to submit your research? Choose BMC and benefit from:

- fast, convenient online submission

- thorough peer review by experienced researchers in your field

- rapid publication on acceptance

- support for research data, including large and complex data types

- gold Open Access which fosters wider collaboration and increased citations

- maximum visibility for your research: over $100 \mathrm{M}$ website views per year

At $\mathrm{BMC}$, research is always in progress.

Learn more biomedcentral.com/submissions 